\title{
Scaling Laws for Weakly-nonlinear WDM Dispersion Managed OOK Systems
}

\author{
P. Serena, A. Orlandini, A. Bononi
}

Università degli Studi di Parma, Parco Area delle Scienze 181/A, 43100 Parma (Italy), email: serena@tlc.unipr.it

Abstract We show that kerr-induced nonlinear effects in long-haul dispersion mapped WDM systems follow general scaling laws that quickly allow both finding the best dispersion map and setting up accurate numerical simulations.

\section{Introduction}

The performance of an optical wavelength-division multiplexed (WDM) transmission system in the nonlinear regime depends on self-phase modulation (SPM), cross-phase modulation (XPM), four-wave mixing (FWM), parametric gain (PG), and their interplay with group-velocity dispersion (GVD). The balance among these effects can be optimized by a careful link design through the use of pre-, in-line and postcompensation fibres. The numerical optimization of system performance is a very time-consuming task, which is usually carried out by introducing several approximations which neglect part of these effects. In this paper, we address the problem of finding general scaling rules that help the designer to i) identify systems with equal performance ii) setup the numerical parameters for a correct and fast simulation. To this aim, we study a WDM system with on-off-keying (OOK) modulation and non-return to zero (NRZ) pulses over a wide range of bit rate, power and spectral efficiency, showing how they can be combined into simple scaling rules.

\section{Theory}

It has been shown in [1] that an ultra-long periodic dispersion-mapped system, having $N$ spans of $L \mathrm{~km}$ each, with transmission fibre attenuation $\alpha$, nonlinear coefficient $\gamma$ and dispersion coefficient $D$, can be described by the dispersion-managed nonlinear Schrödinger equation (DMNLSE). For a single channel $A(z, t)$ normalized to its peak power $P_{\text {peak }}$, the DMNLSE can be written in integral form as:

$$
\begin{aligned}
& \frac{\partial A}{\partial z}=\frac{j}{2 L_{D}} \frac{\partial^{2} A}{\partial t^{2}}-\frac{j|S|}{L_{N L}} \iint r\left(t_{1} t_{2}\right) \times \\
& A\left(t+\sqrt{|S|} t_{1}\right) A\left(t+\sqrt{|S|} t_{2}\right) A^{*}\left(t+\sqrt{|S|}\left(t_{1}+t_{2}\right)\right) d t_{1} d t_{2}
\end{aligned}
$$

where $t$ is the retarded time normalized to $d / R, R$ being the bit rate and $d$ the duty cycle; $L_{N L}=\alpha L /\left(\gamma P_{\text {peak }}\right)$ is the span-averaged nonlinear length; $L_{D}$ is the spanaveraged dispersion length, which can be derived from the normalized in-line dispersion $\xi_{\text {in }}=-N \cdot L / L_{D}=$ $\chi D_{\text {in }}$, being $D_{\text {in }}[\mathrm{ps} / \mathrm{nm}]$ the in-line dispersion cumulated after $N$ spans. The normalized-to-standard conversion factor is $\chi=(\lambda R / d)^{2} /(2 \pi c)$, with $\lambda$ the channel wavelength and $c$ the speed of light. We call $S=$ $\chi \Delta D / \alpha$ the strength of the terrestrial map [2], proportional to the deviation of the transmission fibre dispersion $D$ from its average, i.e. $\Delta D=D-D_{\text {in }} /(N L)$. The fibre kernel $r\left(t_{1} t_{2}\right)$ has two-dimensional Fourier transform $r\left(\omega_{1} \omega_{2}\right)=\left(1+j \operatorname{sgn}(S) \omega_{1} \omega_{2}\right)^{-1}$, being $\omega_{1,2}$ an- gular frequencies. The DMNLSE reveals that the nonlinear effect at time $t$ depends on the signal over a memory time window that scales with $\sqrt{|S|}$. Moreover, for small $S$, the SPM effect scales with the ratio $|S| / L_{N L}$. The XPM effect can be well described by the simple model in [3], in which the XPM distortion is obtained as a filtering of the interfering WDM channels. Here we generalize such model to our ultra-long case by including XPM in (1) and by exploiting a linearization of the interference perturbation as in [3]. Below we report, for the first time, a closed-form expression of the XPM filter $H_{X}$ that relates the received optical field distortion to the AC power of an interfering channel, placed $p$ times the channel spacing $\Delta f[\mathrm{~Hz}]$ to the left of the reference channel:

$$
\begin{aligned}
H_{X}(\omega)= & \frac{-2 j \Phi_{N L} e^{-j \frac{\xi_{i n}}{2} \frac{2 \pi p}{\eta} \omega} e^{j \frac{\omega^{2}}{2}\left(\frac{\xi_{\text {in }}}{2}+\xi_{\text {post }}\right)}}{1+j S\left[\frac{\omega^{2}}{2}+\frac{2 \pi p}{\eta} \omega\right]} \times \\
& \operatorname{sinc}\left[\frac{\xi_{\text {in }}}{2 \pi}\left(\frac{\omega^{2}}{2}+\frac{2 \pi p}{\eta} \omega\right)\right]
\end{aligned}
$$

where, similarly to $\xi_{\text {in }}$, we introduced the normalized cumulated dispersion $\xi_{\text {post }}$ of a post-compensating fibre placed right before the receiver. $\Phi_{N L}=E_{b} N L / L_{N L}$ is the average cumulated nonlinear phase, being $E_{b}$ the normalized average energy per bit. $\eta=(R / d) / \Delta f$ $[\mathrm{bit} / \mathrm{s} / \mathrm{Hz}]$ is the WDM system spectral efficiency. The closed form expression (2) allows to gain physical insight into the XPM impact. For instance, the number of interfering channels that must be accounted for in a fair simulation with XPM scales with the bandwidth of (2). As a worst case with $\xi_{\text {in }}=0$, the bandwidth scales almost as $\eta /|S| . H_{X}$ is also used to estimate the XPM crosstalk variance [3].

\section{Numerical results}

We simulated a $20 \times 100 \mathrm{~km}$ NRZ-OOK system with $D=8 \mathrm{ps} / \mathrm{nm} / \mathrm{km}$, slope $0.058 \mathrm{ps} / \mathrm{nm}^{2} / \mathrm{km}, \gamma=1.7$ $\mathrm{W}^{-1} \mathrm{~km}^{-1}$, and $95 \%$ of in-line dispersion compensation. Before and after the link, pre- and post-dispersion compensating fibres have been inserted to optimize the performance. Such an optimization has been done by exhaustive numerical simulation for each tested setup, i.e. for each bit rate, power and spectral efficiency. At the receiver, a 6th order Butterworth filter of bandwidth $B_{o}=1.8 R$ for $\eta \leq 0.5$, and $B_{o}=1.5 R$ otherwise, selects the central channel of a very large WDM comb. The scaling properties of the nonlinear effects have been investigated by testing different bit 


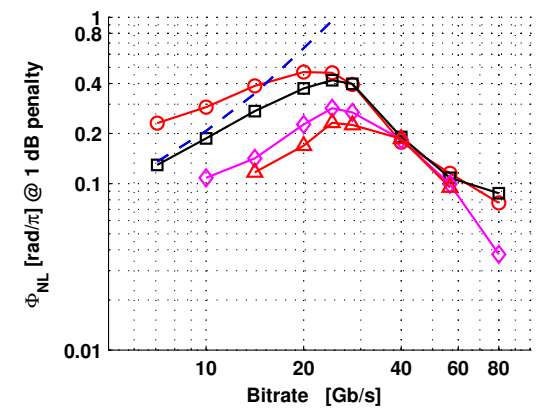

Fig. 1: Nonlinear phase threshold $\Phi_{N L} @ 1 d B$ of OSNR penalty vs. Bit rate R. "o": spectral efficiency $\eta=0.2 ; \square " \eta=0.4 ; " \diamond " \eta=0.6$; " $\triangle " \eta=0.8$. Dashed line: threshold from the XPM filter (2).

rates in the range $7 \div 80 \mathrm{~Gb} / \mathrm{s}$, and different spectral efficiencies in the range $\eta=0.1 \div 0.8$. As a reference starting point in simulations, we verified that at $R=10$ $\mathrm{Gb} / \mathrm{s}$, and $\eta=0.4,19$ channels and 64 bits/ch were enough to accurately evaluate the OSNR penalty in the presence of cross-channel nonlinearities. Then, for the other values of $R$ and $\eta$ we scaled the simulated number of bits with $\sqrt{|S|}$ and the number of channels with $\eta /|S|$, as observed in the previous section. The last rule is a conservative worst case bound for XPM. The Nyquist frequency was chosen to capture at least FWM between the reference and the last side channel.

Fig. 1 shows in a log-log scale the simulated $\Phi_{N L}$ that gives $1 \mathrm{~dB}$ of OSNR penalty vs. back-to-back at $B E R=10^{-5}$. At small bit rates the curves spread because of the XPM effect depending on the spectral efficiency, while at large bit rates, where XPM has disappeared, the curves tend to merge, being affected only by SPM. The dashed line in the figure shows the corresponding $\Phi_{N L}$ predicted from (2) along the lines in [4] (only the $\eta=0.4$ case is shown for clarity). The good match between analytical and numerical results is also a good indicator that FWM is negligible in this case study. When SPM dominates, the threshold is $\Phi_{N L} \propto 1 /|S| \propto 1 / R^{2}$, i.e. it has slope -2. From the plot we infer that, for a given spectral efficiency $\eta$, there exists an optimal transmission fibre dispersion length (i.e. a product $D R^{2}$ ) for which the penalty of XPM and SPM are the same. For the case under investigation with $D=8 \mathrm{ps} / \mathrm{nm} / \mathrm{km}$ the best bit rate varies in the range $15 \div 30 \mathrm{~Gb} / \mathrm{s}$ by varying $\eta$.

Fig. 2 depicts OSNR penalty versus the product $\Phi_{N L} \eta$ at $R=10 \mathrm{~Gb} / \mathrm{s}$ and $R=20 \mathrm{~Gb} / \mathrm{s}$ by varying $\Phi_{N L}=$ $0.1 \pi \div 0.6 \pi$ and $\eta=0.4 \div 0.7$. Here we optimized the entire link, including in-line dispersion, for each $\Phi_{N L}$ and $\eta$. The solid lines represent a best fit of the measured points showing that the XPM penalty, here dominant, indeed scales with the product $\Phi_{N L} \eta$.

Fig. 3 shows the $1 \mathrm{~dB}$ nonlinear threshold vs. strength in a very large WDM comb when SPM, XPM, FWM and noise $P G$ act individually. Here we fixed the normalized in-line dispersion to $\xi_{\text {in }}=1.5 \cdot 10^{-3}$, while we set the post-compensation to either $\xi_{\text {post }}=2.5 \cdot 10^{-3}$

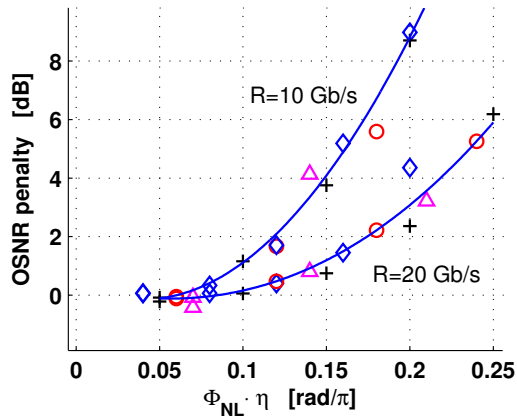

Fig. 2: OSNR penalty vs. the product $\Phi_{N L} \eta$. " $\diamond ": \eta=$ $0.4 ; "+": \eta=0.5 ; "$;": $\eta=0.6$; " $\triangle ": \eta=0.7$.

(dashed lines) or $\xi_{\text {post }}=5 \cdot 10^{-3}$ (solid lines). Precompensation was equal to zero. The penalty of XPM was evaluated from (2) as before, the penalty of FWM was obtained using the standard undepleted-pump approximation, and the threshold due to $P G$ was shown in [2] to scale as $\Phi_{N L} \propto|S|^{1 / 8}$ and to decrease with OSNR. It can be shown that the FWM nonlinear phase scales as $\Phi_{N L} \propto|S| / \eta^{2}$, while the XPM nonlinear phase as $\Phi_{N L} \propto|S| / \eta$ when $S$ is large enough. In the figure, note the opposite impact of post-compensation on XPM and SPM, which moves the optimal strength to the right for increasing $\xi_{\text {post }}$, while FWM is basically unaffected. Finally, as the strength increases from zero, one can clearly understand from this figure that the dominant impairment is first FWM, then XPM, then $P G$ (if the OSNR is sufficiently small) and lastly SPM.

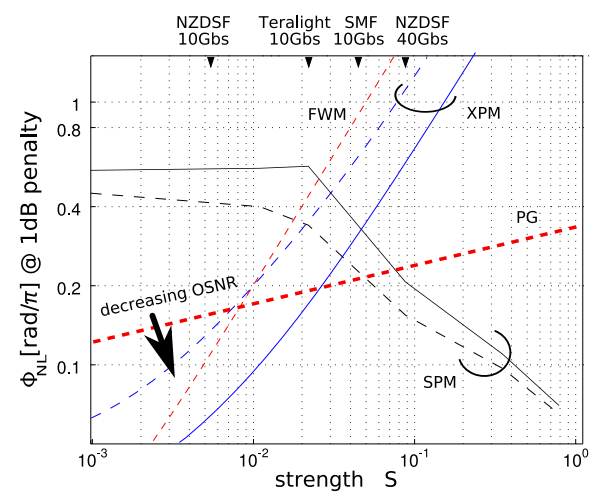

Fig. 3: SPM, XPM, FWM, and PG nonlinear thresholds $\Phi_{N L} @ 1 d B$ of OSNR penalty vs. S. Dashed: $\xi_{\text {post }}=$ $2.5 \cdot 10^{-3}$. Solid: $\xi_{\text {post }}=5 \cdot 10^{-3} \cdot \eta=0.2$.

\section{Conclusions}

We clarified how nonlinear penalties in long-haul WDM dispersion mapped systems with OOK modulation scale with the main system parameters, namely map strength, nonlinear phase and spectral efficiency, thus showing the regions of operation in which the individual effects of FWM, XPM, PG and SPM dominate.

\section{References}

1. M. J. Ablowitz et al., JOSAB, v. 19, 425-439 (2002).

2. P. Serena et al., JLT, v. 24, to appear, May 2006.

3. G. Bellotti et al., PTL, v. 10, 1745-1747 (1998).

4. R. S. Luis et al., JLT, v. 23, 1503-1513 (2005). 